Journal of Computer Science 3 (11): 887-893, 2007

ISSN 1549-3636

(C) 2007 Science Publications

\title{
Theoretical and Simulation Approaches for Studying Compensation Strategies of Nonlinear Effects Digital Lightwave Links Using DWDM Technology
}

\author{
J. Bel Hadj Tahar \\ Graduate School of communications of Tunis, Sup'Com \\ El Ghazala Pole of Technology, 2083 Ariana, Tunisia
}

\begin{abstract}
Nowadays, techniques for education are based on the combination of theoretical formulas, experimental measurement and simulation approaches. Theoretical analysis, experimental and simulation approaches based educations allow developing simple and non expensive tools for the aim of studying complex problems in optical network communication. Theoretical formulas, used to describe the non linear effects on digital lightwave links, may by simplified with the use of simulation models so that real and industrial applications can be approached. In this study, we use theoretical model taking into account system parameters, physical penalty and transmission speed, for the purpose of developing simulation tools. Using these simulation techniques, it is possible to investigate various compensation techniques and demonstrate their effects on system performances.
\end{abstract}

Key words: Theoretical analysis, simulation techniques, compensation techniques, transmission optical system, DWDM technology

\section{INTRODUCTION}

Rapid growth of the global communication network is being made possible by the availability of new optical fiber technologies. Dense Wavelength Division Multiplexing system DWDM saves the network operators enormous amounts of money in the process of upgrading the network capacities, although the technology itself is complex and expensive. With the recent developments in digital lightwave links and the realization of multi-gigabit transmission systems employing DWDM, the study of impact of nonlinear transmission effects, such self-phase modulation (SPM) and cross-phase modulation (XPM), four wave mixing $(\mathrm{FWM}), \ldots$, is of a significant importance ${ }^{[1,4,5]}$. Unfortunately, modeling and theoretical analysis of DWDM system are interesting but difficult, because it requires many areas of knowledge such physical theory of optical dispersion, as well as laser and photo detector conversion phenomena and models. Alternately, the simulation technique is a quite tool that can be used to simplify this problem and to approach, in the same time, experimental and realistic cases. Therefore, computer simulation plays an important role in the design, optimization and evaluation of system performances in the presence of nonlinear distortions. It is obvious that the complexity of the experimental system increases with that with of the communication system.
Basically, a DWDM system is composed of an emitter module, single mode fiber, multiplexer and demultiplexer component, erbium-doped fiber amplifier and receiver bloc. For every optoelectronic component used, a Matlab/Simulink model is developed and tested in this work.

As well, in the evaluation of system performance, Matlab/Simulink is commonly used to study the effects of nonlinear distortion on the transmission capacity of DWDM based optical communication networks. To analyze introduced nonlinear distortion, simplified model function is developed and ameliorated to approach progressively experimental response. The chromatic dispersion penalty due to the information bandwidth can be evaluated only from the electrical field domain model. An examination of the effect of system characteristics variations is shown in terms of BER and eye diagram analysis.

\section{MODELLING AND SIMULATION OF DWDM SYSTEM}

Transmitter bloc: The basic of an optical transmitter bloc involves a signal generator, a semiconductor laser and an optical multiplexer $\mathrm{N}: 1$. The mixer output is transmitted through a single optical fiber.

The input to the optical source is represented by the coded output of a pseudorandom data generator with binary values 0 and 1 . The word lengths are sufficient 
to analyze nonlinear effects. Generally, non return to zero (NRZ) and return-to- zero (RZ) are the leading coding used in light wave communication. Other form of signal may be used, the transmission bit rate and signal level are defined using Matlab/Simulink parameter. Different signal characteristics are tested with modified intensity and bit rate level.

The important characteristics of optical laser are the optical spectrum, the temporal response defined by the rise time and the fall time and the waveform describing the oscillation mode of the laser. When the laser is modulated directly by the excitation courant, the carrier density is not clamped at the threshold value. The modulation of carrier density is accompanied by a modulation of the index of refraction and that to frequency modulation. A expression of the laser frequency chirp is defined by ${ }^{[1]}$.

$$
\Delta \lambda(\mathrm{t})=\frac{-\alpha}{4 \pi}\left[\frac{1 \mathrm{dP}}{\mathrm{P}_{0} \mathrm{dt}}+\frac{\varepsilon}{\tau \mathrm{p}} \Delta \mathrm{P}(\mathrm{t})\right]
$$

where $\alpha$ is a phase amplitude coupling, $\varepsilon$ is the gain suppression factor and $\tau_{\mathrm{p}}$ is the photon lifetime. So, optical laser is modeled by two filters and a delay time given by :

$$
t_{d}=\tau_{n} \operatorname{In}\left(\frac{I_{p}-I}{I_{s}-I}\right)
$$

where $\tau_{\mathrm{n}}$ is the carrier lifetime, $\mathrm{I}_{\mathrm{p}}$ is the polarisation current and $I_{\mathrm{s}}$ is the threshold current.

Using differential equations describing laser behavior, the related transfer function can be written as.

$$
\begin{aligned}
& T(\omega)=\frac{P(\omega)}{I(\omega)} \\
& =\frac{\frac{A\left(1-\varepsilon P_{0}\right) P_{0}}{e}}{\omega^{2}+\frac{\varepsilon P_{0}+A \tau_{n}\left(1-\varepsilon P_{0}\right)^{2} P_{0}}{\tau_{n} \tau_{p}\left(1-\varepsilon P_{0}\right)}+\omega\left(\frac{1}{\tau_{n}}+\frac{\varepsilon P_{0}+A \tau_{p}\left(1-\varepsilon P_{0}\right)^{2} P_{0}}{\tau_{p}\left(1-\varepsilon P_{0}\right)}\right)}
\end{aligned}
$$
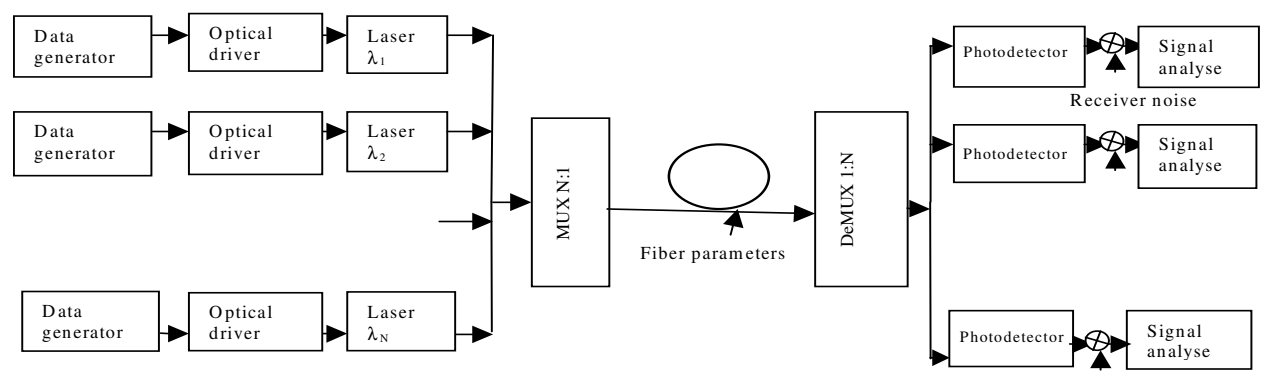

Fig. 1: Block diagram of digital light wave transmission system using DWDM technique 888
Where $A$ is the differential gain coefficient and $\varepsilon$ is compression factor.

This function can be written as :

$$
\mathrm{T}(\mathrm{p})=\frac{\alpha}{\mathrm{p}^{2}+\alpha \mathrm{p}+\beta}
$$

represented with second order filter.

The laser chirping model is described by an additive functions to $F_{1}(t)$ and $F_{2}(t)$ in Fig. 1.

Student concentrates to find the dependence between physical models and simulated ones. They often study the effect of laser physical parameters on output signal characteristics when a high bit-rate, more then $2.5 \mathrm{Gbit} / \mathrm{s}$, is transmitted. An example of Matlab/Simulink model of laser source is given in Fig. 2. Comparing output signals of laser with the experimental response shows the validity of this equivalent circuit model.

Optical fiber modeling: Only single mode SM fiber is considered. Its model should take into account the attenuation and dispersion effects. The base band transfer function of the single mode fiber, in the power domain, is given by ${ }^{[2]}$.

$$
H(f)=\int_{-\infty}^{+\infty} \sigma(\lambda) \cdot \alpha(\lambda) \cdot e^{-j \omega T(\lambda)} d \lambda
$$

where $\sigma(\lambda)$ is the source spectrum versus wavelength, $\alpha$ $(\lambda)$ is the fiber attenuation for length $\lambda$ versus wavelength, $T(\lambda)$ is the fiber group delay per unit length.

$$
\frac{\mathrm{dT}(\lambda)}{\mathrm{df}}=\mathrm{D}(\lambda) \frac{\lambda^{2}}{\mathrm{c}}
$$

$\mathrm{D}(\lambda)$ is the chromatic dispersion.

If we take into account introduced Kerr and Raman nonlinear effects, the evolution equation of optical power is described by nonlinear equation of Schrödinger ${ }^{[4]}$. 


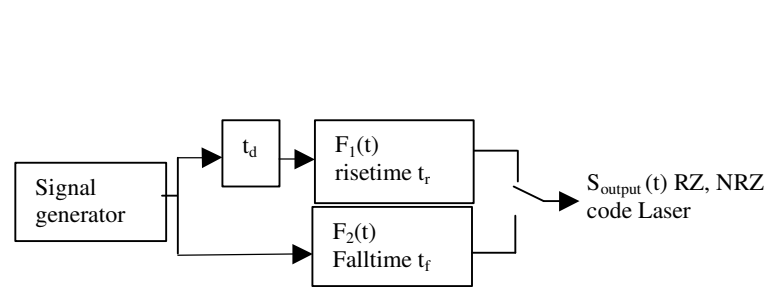

(a)

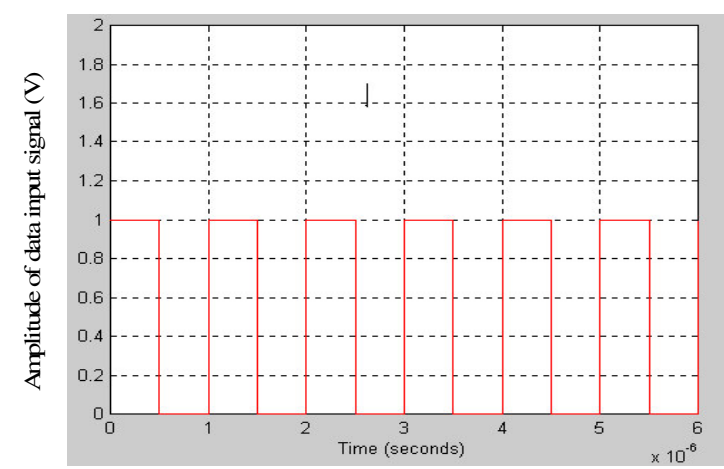

(c)

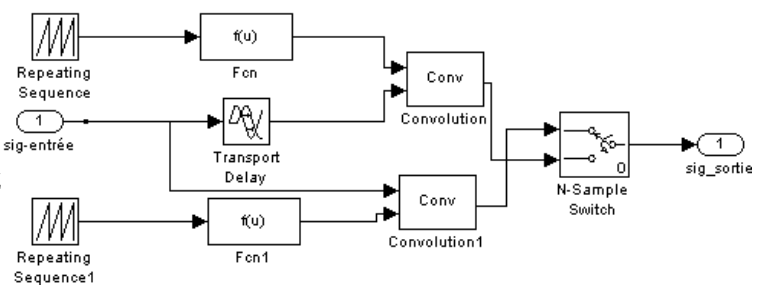

(b)

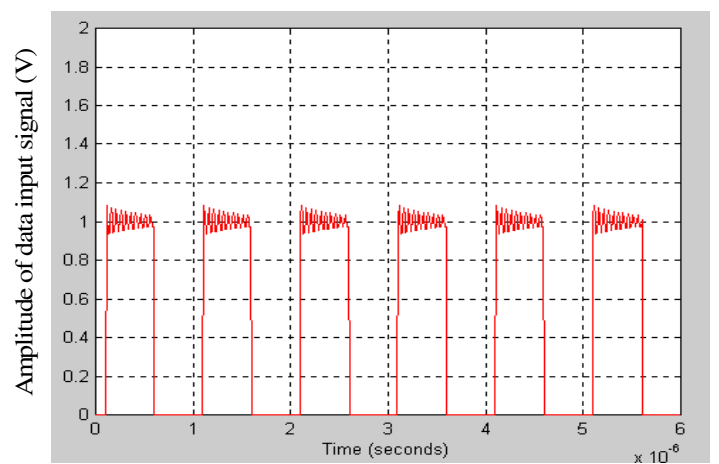

(d)

Fig. 2 : The time evolutions of signal channel powers at the input and output ends of laser diode, (a) block diagram, (b) Simulink model and (c) Data input signal and d: temporal signal at Laser output

$\frac{\partial \mathrm{A}(\mathrm{z}, \mathrm{t})}{\partial \mathrm{z}}=-\frac{\alpha}{2} \mathrm{~A}(\mathrm{z}, \mathrm{t}):$ exp rime the linear attenuation,

$\mathrm{Z}:$ is the dis tan ce and is the attenuation coefficient

$-\mathrm{j} \frac{\beta_{2}}{2} \frac{\partial^{2} \mathrm{~A}(\mathrm{z}, \mathrm{t})}{\partial^{2} \mathrm{t}}$ : exp rime the sec ond order dispersion term,

$\beta_{2}$ : is the sec ond order propagation constant $\left(\mathrm{ps}^{2} \mathrm{~km}^{-1}\right)$

$+\mathrm{j} \frac{\beta_{3}}{2} \frac{\partial^{3} \mathrm{~A}(\mathrm{z}, \mathrm{t})}{\partial^{3} \mathrm{t}}$ :exp rime the therd order dispersion term,

$\beta_{3}$ : is the sec ond order propagation constant $\left(\mathrm{ps}^{3} \mathrm{~km}^{-1}\right)$

$+\mathrm{j} \gamma|\mathrm{A}(\mathrm{z}, \mathrm{t})|^{2} \mathrm{~A}(\mathrm{z}, \mathrm{t}) \quad$ : exp rime Kerr effect, $\gamma:$ is the non li nearity coefficient

$+\mathrm{j} \gamma \mathrm{T}_{\mathrm{R}} \frac{\partial}{\partial \mathrm{t}}|\mathrm{A}(\mathrm{z}, \mathrm{t})|^{2} \mathrm{~A}(\mathrm{z}, \mathrm{t}):$ exp rime the Raman effect

$-\frac{\gamma}{\omega_{0}} \frac{\partial}{\partial \mathrm{t}}|\mathrm{A}(\mathrm{z}, \mathrm{t})|^{2} \mathrm{~A}(\mathrm{z}, \mathrm{t})$ : exp rime self steepening effect,

$$
\omega_{0}: \text { is the angular frequency }
$$

Then SM fiber is modeled with a function bloc allowing its temporal dependencies with wavelength, bit rate and chromatic dispersion.

The basic model of single mode fiber can introduce the attenuation term to which we can add other bloc functions describing the 2nd and 3rd order linear effects. Bloc functions are developed using simulink filter model describing the comportment of various terms to approach nonlinear optical effects defined in the Eq. 7. For some examples, where, the transmission speed is low, the number of channels is reduced and the distance is short, the second terms can be neglected. For a high data rate and a high power level, the non linear effects have to be taken into account; we should add that, the model complexity increases when the propagation effects are considered. Fig. 3 gives an example of the influence of nonlinear parameters on the time variations of transmitted signal on SM optical fiber.

Receiver bloc: The receiver bloc is composed a PIN photosensitive detector or avalanche photo detector. Electrical model should take into account the shot and thermal noise. So, that receiver is modeled as a filter with additive Gaussian noise at the input. This simulation shows that the effect of photo detector excess noise is to influence the BER performance of the receiver. Simulink library gives varying noise models. The PIN and APD diodes have been simulated using time domain models. A Gaussian approximation for the shot noise has been implemented using the approach discussed $i^{[2]}$. In different cases, students have accounted for the shaping of the signal, the added thermal noise and the possibility that the noise is white or colored. A colored noise can be simulated by properly filtering (Fig. 4). 


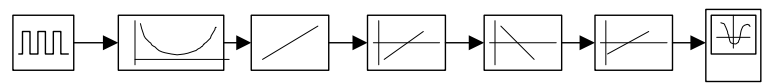

(a)

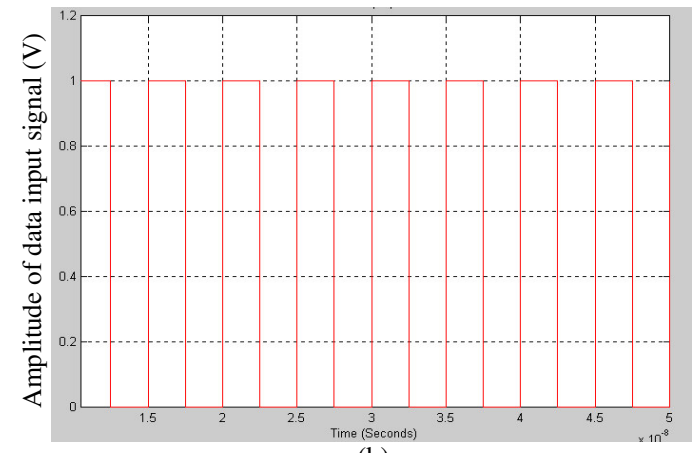

(b)

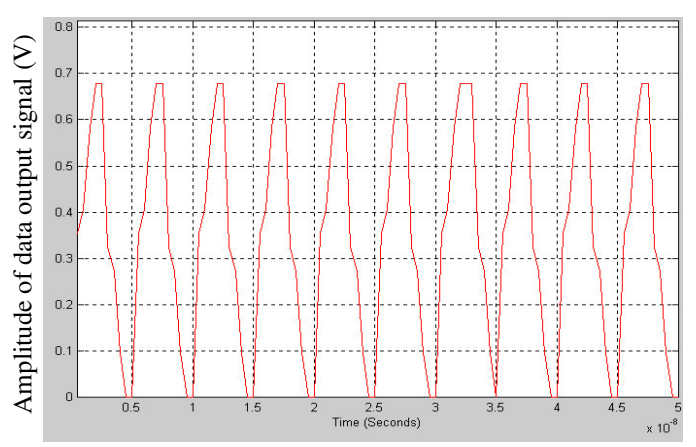

(c)

Fig. 3: The time evolutions of signal channel powers at the input and output ends of SM optical fiber, (a) Simulink model, (b) Data input signal and (c) temporal signal at fiber output (fiber length $20 \mathrm{Km}, \mathrm{D}=1 \mathrm{ps} / \mathrm{nm} . \mathrm{Km}$, attenuation $0.2 \mathrm{~dB} \mathrm{Km}^{-1}$ )

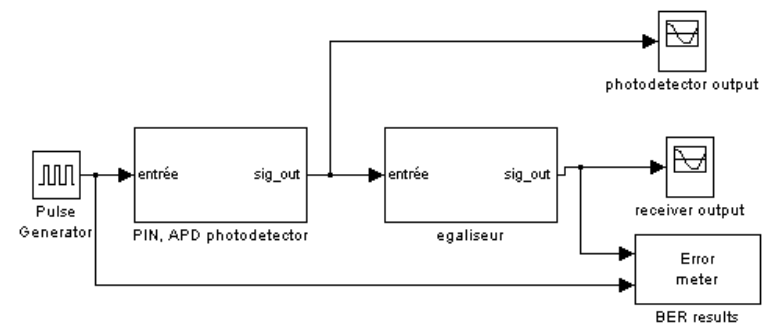

(a)

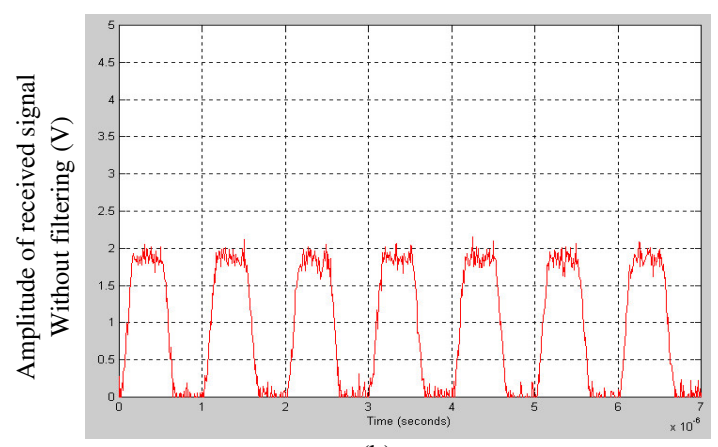

(b)

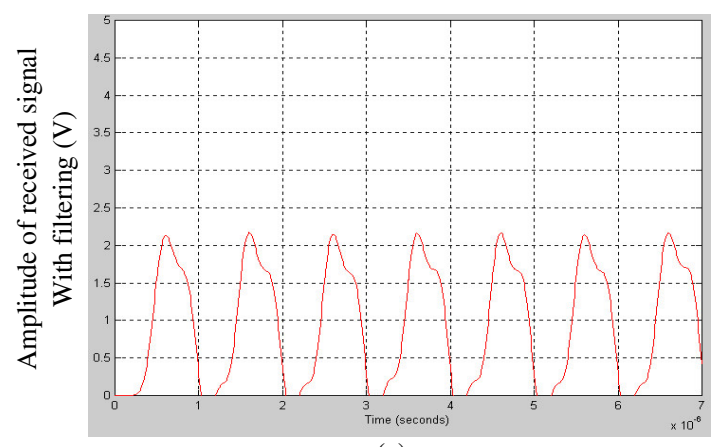

(c)

Fig. 4: APD receiver performance, (a) Simulink model, (b) temporal signal at receiver output without filtering and (c) temporal signal at receiver output with filtering

To minimize system noise, we have to give solutions by using adaptive filter and amplifier placed after the photo detector. Different blocs are tested separately to evaluate there temporal and spectral responses.

With the combination of all bloc functions composing the optical transmission system it is possible to evaluate the maximum transmission distance for defined parameters. In the same way, the student can obtain the plots of the temporal response or the eye diagram at the receiver output. On the other side, we can imagine solutions allowing to reduce these non linear effect by the addition of bloc functions.

The difficulty in this study is to find a good approach between physical parameters and electrical model. Sample results of simulation employing DWDM 


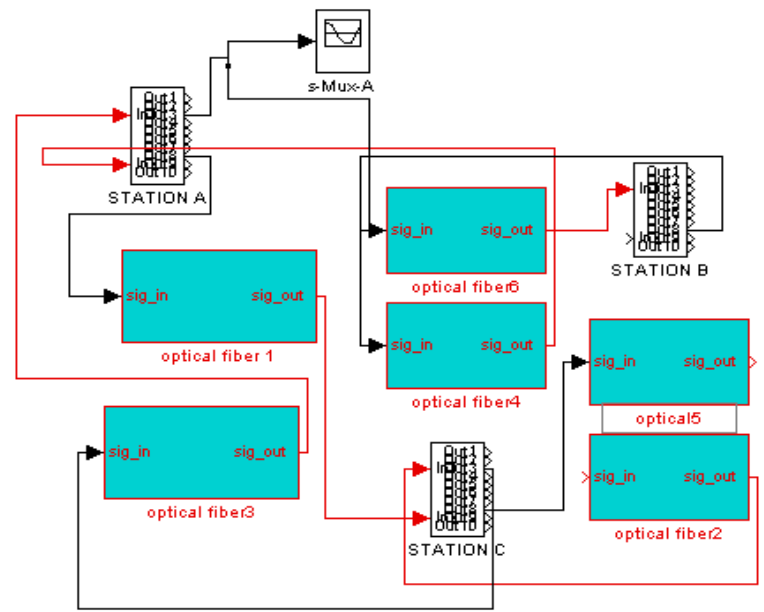

(a)

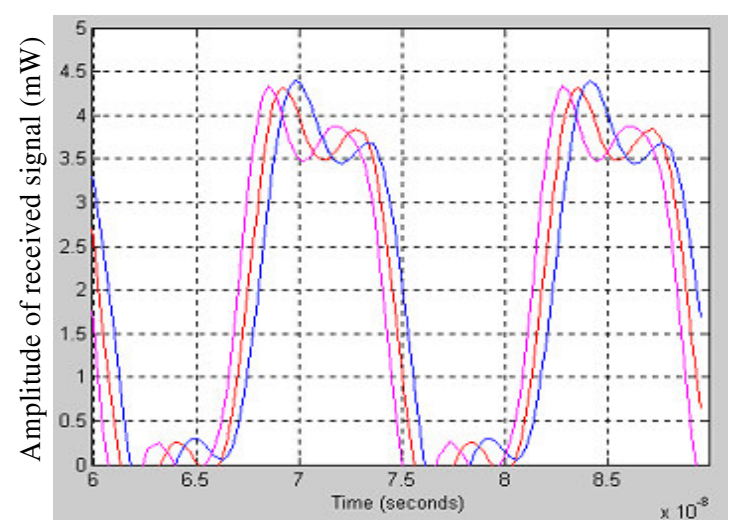

(b)

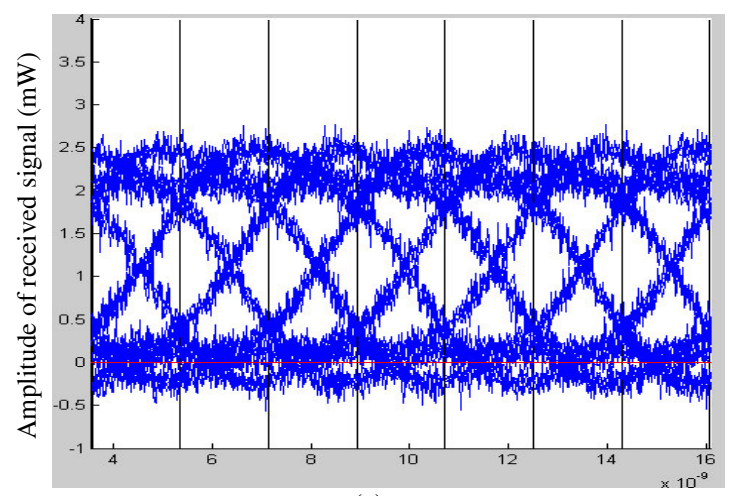

(c)

Fig. 5: Digital light wave system performance, (a) Simulink model, (b) temporal signal at receiver output(multiplexed optical signal $\mathrm{N}: 3$ ) and (c) Eye diagram (after demultiplexing) for $2.5 \mathrm{Gbit} / \mathrm{s}$ transmission over $100 \mathrm{Km} \mathrm{SM}$ fiber, D = 1 ps/nm. Km (station A)

technique are discussed. A basic end-to-end single mode digital light wave system is analyzed for different transmitted capacity of $140 \mathrm{Mbit} / \mathrm{s}, 2.5,10$ and 40 Gbit/s Fig. 5 studies an example of digital light wave system composed of three stations and three transmitting $(\mathrm{n}=3)$ wavelengths.

\section{PERFORMANCE EVALUATION OF DWDM SYSTEMS WITH AND WITHOUT COMPENSATION}

The transmission penalty, due to dispersion induced self-phase modulation and cross-phase modulation, in wavelength division multiplexing systems has been evaluated following a matrix analysis. The transmission system is composed of fiber of length $\mathrm{L}$, which is divided into $\mathrm{N}$ segments such that every segment is represented with a bloc function.
Considering the input-output column vectors, the power fluctuation part of the signal of p-th segment in a WDM system can be put in the following form ${ }^{[6]}$ :

$\left[\begin{array}{c}S^{1}(p+1) \\ S^{2}(p+1) \\ \ldots \ldots \\ S^{k}(p+1) \\ \ldots \ldots \\ S^{\mathrm{M}}(p+1)\end{array}\right]=\left[\begin{array}{cccccc}\mathrm{A}_{\mathrm{s}}^{1}(\mathrm{p}) & \mathrm{A}_{\mathrm{x}}^{2}(\mathrm{p}) & \ldots & \ldots & \ldots & \mathrm{A}_{\mathrm{x}}^{\mathrm{M}}(\mathrm{p}) \\ \mathrm{A}_{\mathrm{x}}^{2}(\mathrm{p}) & \mathrm{A}_{\mathrm{s}}^{2}(\mathrm{p}) & \ldots & \ldots & \ldots & \ldots \\ \ldots & \ldots & \ldots & \ldots & \ldots & \ldots \\ \mathrm{A}_{\mathrm{x}}^{\mathrm{k}}(\mathrm{p}) & \ldots & \ldots & \mathrm{A}_{\mathrm{s}}^{\mathrm{k}}(\mathrm{p}) & \ldots & \mathrm{A}_{\mathrm{x}}^{\mathrm{k}}(\mathrm{p}) \\ \ldots & \ldots & \ldots & \ldots & \ldots & \ldots \\ \mathrm{A}_{\mathrm{x}}^{\mathrm{M}}(\mathrm{p}) & \ldots & \ldots & \ldots & \ldots & \mathrm{A}_{\mathrm{s}}^{\mathrm{M}}(\mathrm{p})\end{array}\right] *\left[\begin{array}{c}\mathrm{S}^{\mathrm{l}}(\mathrm{p}) \\ \mathrm{S}^{2}(\mathrm{p}) \\ \ldots \ldots \\ \mathrm{S}^{\mathrm{k}}(\mathrm{p}) \\ \ldots \ldots \\ \mathrm{S}^{\mathrm{M}}(\mathrm{p})\end{array}\right](8)$

where, $\mathrm{k}=1,2 \ldots \mathrm{M}$ is a channel number in a $\mathrm{M}$ channels WDM system and $\mathrm{p}=1,2, \ldots \mathrm{N}$ is a segment number. Also, $A_{s}{ }^{k}$ is the self-phase modulation (SPM), given by ${ }^{[6]}$ and $A_{x}{ }^{k}$ while stands for the cross-phase modulation (XPM). 


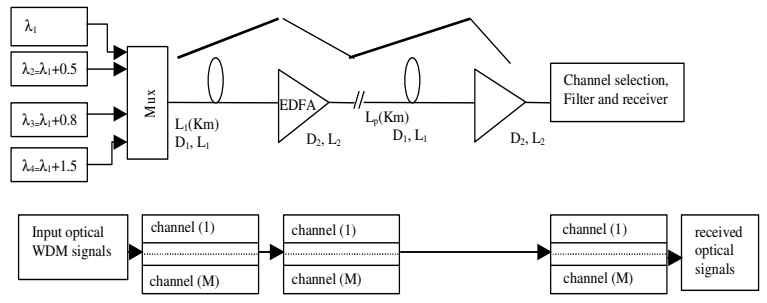

Fig. 6: Dispersion map for Standard Single mode fibre (SSMF) $\mathrm{D}_{1}=17 \mathrm{ps} / \mathrm{nm}$. Km and Dispersion compensating fibre DCF, $\mathrm{D}_{2}=-85 \mathrm{ps} / \mathrm{nm}$. Km, $\mathrm{L}_{1}=40 \mathrm{Km}, \mathrm{L}_{2}=10 \mathrm{Km}$

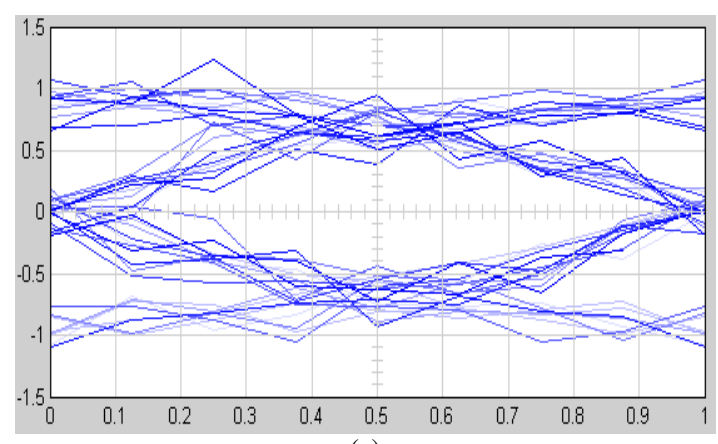

(a)

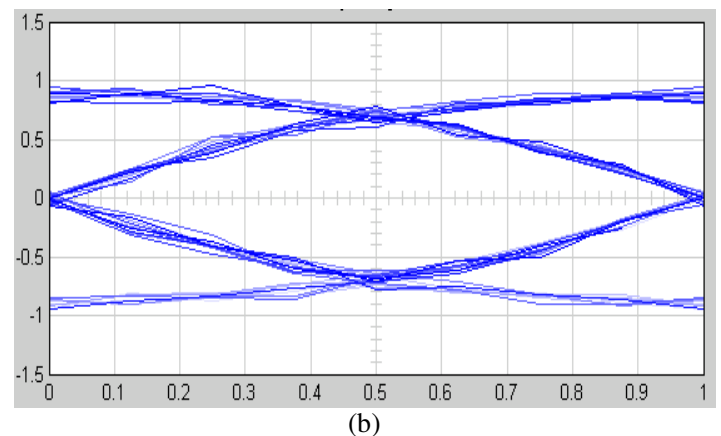

(b)

Fig. 7: (a) eye diagram of output signal of a transmission optical system (of best channel) without compensation using only standard fibre SSMF and (b) using compensating map of a transmission optical system composed on $\mathrm{N}=4$ segments, $\mathrm{L}_{1}=\mathrm{L}_{3}=40, \mathrm{~L}_{2}=\mathrm{L}_{4}=10 \mathrm{Km}$, $\mathrm{D}_{1}=\mathrm{D}_{3}=17 \mathrm{ps} / \mathrm{nm} . \mathrm{Km} \quad(\mathrm{SSMF})$, $\mathrm{D}_{2}=\mathrm{D}_{4}=-100 \mathrm{ps} / \mathrm{nm} . \mathrm{Km}(\mathrm{DCF})$

In the simulation technique, each channel defined with $\mathrm{A}_{\mathrm{s}}{ }^{\mathrm{k}}(\mathrm{p})$ term is represented with a bloc function, where dispersion parameters and fiber characteristics are introduced (Fig. 6).

The dispersion map is used to reduce the impact of nonlinearities. Different solutions are used as standard single-mode fiber $(\mathrm{SSMF})+(\mathrm{SSMF})$, dispersion

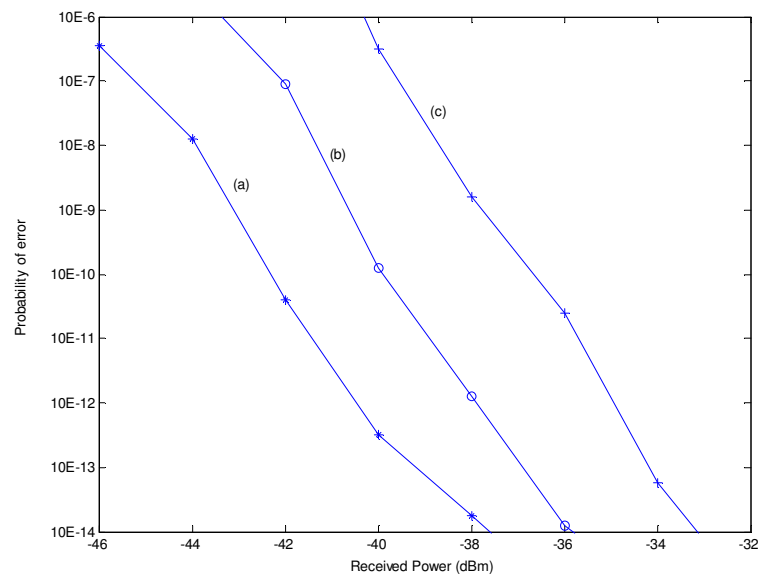

Fig. 8: (a and b) BER curve for the 1st channel (best) and 4th channel (worst) and (c) 1st channel without compensation

compensating fiber $(\mathrm{DCF})+(\mathrm{SSMF}), \ldots$ and different spans of $\mathrm{L}(\mathrm{Km})$ are analysed to evaluate the importance of different compensation techniques. Figure 7 and 8 give examples of simulation results and show the importance place that take compensation techniques to reduce the distortions on the received signals. The response of the optical system describe as better realistic cases and experimental measurements of digital optical network.

\section{CONCLUSION}

With the increasing demand for low-cost broadband service, optimizing nonlinear effects on optical communication systems becomes a key to driving down the cost per bit per kilometer. For this purpose, computer simulation plays an important role in the design, analysis and evaluation of DWDM system performance. It allows students to develop more and more complex optical model by means of which optimum results can be provided and interpreted.

When developing a model based on theoretical formulation, one can see the importance of taking into account or neglecting physical parameters. It is easy to study the influence of different parameters on quality of transmission (QoS) in terms of BER and eye diagram and that can simplify complex theoretical expressions. This method is there fore a tool that can be used by student to optimize fiber parameters according to a required performance.

These flexible techniques have to develop theoretical functions and experimental results to describe as better existing optical system response or to optimize future solutions on optical networks and in the 
same time to approach to students physical phenomena affecting the capacity transmission of optical fibers.

\section{REFERENCES}

1. Liu, M.M., 1996. Principe and Application of Optical Communications, Chicago, IL, R.D. Irwin.

2. Elrefaie, A.F., J.K. Townsend, M.B. Romeiser and K.S. Shanmugan, 1988. Computer simulation of digital lightwave links, IEEE J. Selected areas in Communicat., 6 (1): 94-105.

3. Pires, V.F., J. Fernando and A. Silvia, 2002. Teaching Nonlinear Modeling, Simulation and control of electronic power converters using Matlab/Simulink, IEEE Transaction Educat., 45 (3): 253.
4. Midwinter, J.E. and Y.L. Guo, 1995. Optoelecronics and Lightwave Technol., WileyInterscience Publication.

5. Yariv, 1997. Optical Electronics in modern communications, 5th Edn., Ed. Oxford University Press.

6. Pal, B., S.P. Majumdar and R. Gangopadhyay, 1998. Evaluation by matrix method heterodyne CPFSK transmission penality due to GVD, SPM and ASE noise, in Proc IEEE LEOS Conf. Florida, USA.

7. Yamazaki, S., T. Ono and T. Ogata, 1993. Compensation for chromatic dispersion and nonlineair effect, IEEE J. Lightwave Technol., 11: 603-611. 